\title{
Using synthetic forms of RS5 and RS7 to expand the genetic diversity of common wheat for disease resistance
}

\author{
Davoyan R.O. ${ }^{1}$, Bebykina I.V. ${ }^{1}$, Davoyan E.R. ${ }^{1}$, Zubanova Y.S. ${ }^{1}$, Zinchenco A.N. ${ }^{1}$, \\ Badaeva E.D. ${ }^{2}$, Salina E.A. ${ }^{3}$, Adonina I.G. ${ }^{3}$ \\ ${ }^{1}$ National Center of Grain named after P.P. Lukyanenko, Krasnodar, Russia \\ ${ }^{2}$ Vavilov Institute of General Genetics, Russian Academy of Sciences, Moscow, Russia \\ ${ }^{3}$ Institute of Cytology and Genetics, SB RAS, Novosibirsk, Russia \\ * email:davoyanro@mail.ru
}

Synthetic recombination forms RS5 (BBAASD') and RS7 (BBAASU) was obtained from crossing genome substitution form Avrodes (BBAASS) with synthetics T. durum/Ae. tauschii (BBAAD'D ${ }^{t}$ ) and Avrolata (BBAAUU). Resistant to leaf rust, yellow rust and powdery mildew introgression lines have been obtained from backcrosses RS5 and RS7 with the susceptible varieties of common wheat Krasnodarskaya 99 and Rostislav. DNA-analysis of 24 leaf rust resistant lines was carried out for the presence of known effective genes previously identified in synthetic forms: Lr28, Lr35 and Lr51 from Ae. speltoides; Lr9 from Ae. umbellulata and Lr39 from Ae. tauschii. Only one line obtained with the participation of the RS7 synthetic showed presence of the gene Lr28. The cytological study (C-banding and FISH) of the lines obtained using the RS7 form revealed new translocations of T1DS.1DL-1SL, T3DS.3DL-3SL and the substituted chromosome 7D(7S) from Ae. speltoides. New translocations T2DS.2DL-2UL and T7DL.7DS-7US also have been identified from Ae. umbellulata. The new substitutions $4 \mathrm{D}\left(4 \mathrm{D}^{t}\right), 6 \mathrm{D}\left(\mathrm{D}^{\mathrm{t}}\right)$ from Ae. tauschii and $7 \mathrm{D}(7 \mathrm{~S})$ from Ae. speltoides were determined for the first time in the lines obtained from synthetic RS5. Lines with genetic material from two wild species at once were revealed. In the line 3379n14, translocations in chromosomes 5BL, 1DL, 2DL from Ae. speltoides and 7DS from Ae. umbellulata were identified. Line 4626p16 presumably has a 2DS.2DL2UL translocation from Ae. umbellulata and T7SS.7SL-7DL from Ae. speltoides. In the line 4991p17, substituted chromosomes 6D(D) from Ae. tauschii and 7D(7S) from Ae. speltoides were obtained. Presumably, lines with new translocations and substituted chromosomes may carry previously unidentified genes for resistance to fungal diseases, in particular to leaf rust, from the species Ae. speltoides, Ae. umbellulata and Ae. tauschii. 